\title{
Research Paper \\ The Impact of Yoga and Pilates Exercises on Older Adults
}

\author{
${ }^{*}$ Khadije Irandoust ${ }^{1}$, Morteza Taheri ${ }^{1}$ \\ 1. Department of Physical Education, Faculty of Social Sciences, Imam Khomeini International University, Qazvin, Iran.
}

Citation: Irandoust Kh, Taheri M. (2016). [The effects of yoga and Pilates exercises on elder adults (Persian)]. Iranian Journal of Ageing. 2016; 11(4):152-161. http://dx.doi.org/10.21859/sija-1101152

http://dx.doi.org/10.21859/sija-1101152

Received: 12 Nov. 2015 Accepted: 05 Feb. 2016

Key words: Motor performance, Flexibility, Muscular strength, Dynamic balance, Static balance

\section{A B STRACT}

Objectives Old age is a period accompanied by automatic, gradual, and advancing corrosive changes in most organs and physiological systems of the body. One such important change is in the systems involved in balance control, which could expose older people to serious damages, such as bone fractures that are associated with high medical costs, due to poor balance. Therefore, the main purpose of this study was to investigate the effect of yoga and Pilates exercises on motor performance of the elderly people.

Methods \& Materials This study was conducted using a quasi-experimental design. A total of 60 obese subjects (mean [SD] age: 62.2 [1.6] years) were randomly assigned into 3 groups of yoga $(n=20)$, Pilates $(n=20)$, and control $(n=20)$. Yoga practices, which comprised Asana training, Pranaya training, and meditation, were done 3 times a week, for 8 weeks. The Pilates course had 3 sections of warming up, major practices, and cooling down. These practices were done in positions of lying down, sitting, and standing. The movements started from simple activities and gradually got more complicated. Motor performance tests of static and dynamic balance, flexibility, and lower extremity strength were administered. The Stork test was used to evaluate standing balance in terms of seconds and milliseconds. The flexibility was evaluated with Welz test. The test of climbing stairs up and down was used to measure dynamic stability. In this test, the subjects climb up and down 7 times from a $20-\mathrm{cm}$ height or chair. The subject climbs with one leg and then lifts another leg. Next, he or she brings down the first leg and then another leg. Each climbing is considered one time. When the subject did this activity 7 times, the time is recorded. The standing chair test is used to evaluate the muscle strength of lower extremity and legs. While sitting on the edge of the chair, the subject should stand with the best of his or her power in 30 seconds and then returns to sitting position. The mean of number of activities during two 30 -second cycle is regarded as the final record. To analyze the data, we used 1-way ANOVA test and post hoc Tukey test.

Results The results indicated that both Pilates and yoga exercises significantly improved the scores of static balance, dynamic balance, power of lower extremity muscles, and flexibility $(P<0.05)$ while no significant difference was found between 2 experimental groups with regard to improvement in movement performance.

Conclusion According to our research findings, yoga and Pilate's exercises can improve static and dynamic balance and lower extremities strength. Since the improvement in the variables of movement performance has a significant effect on preventing falling of older people, we recommend that these exercises should be followed by the older people.

\section{* Corresponding Author:}

Khadije Irandoust, PhD

Address: Department of Physical Education, Faculty of Social Sciences, Imam Khomeini International University, Qazvin, Iran.

Tel: +98 (912) 2826152

E-mail: parirandoust@gmail.com 


\title{
تأثير يوكا و ييلاتس بر عملكرد حركتى سالمندان
}

\author{
•خديجه ايراندوست'، مرتضى طاهرى' \\ 1- كروه تربيتبدنى، دانشكده علوم اجتماعى، دانشكاه بينالمللى امامخمينى(ره)، قزوين، ايران.
}

\begin{abstract}
حكs

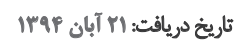
تاريخ بذيرش: 19

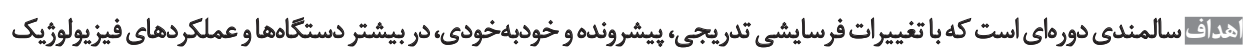

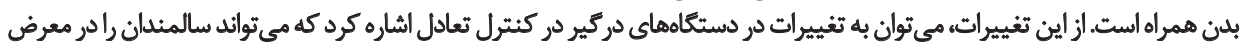

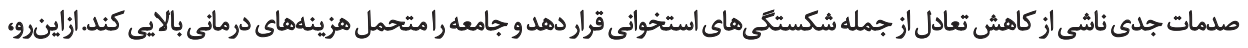

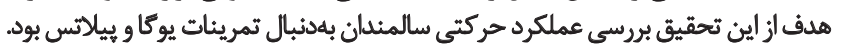

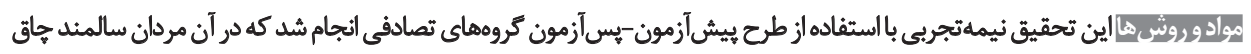

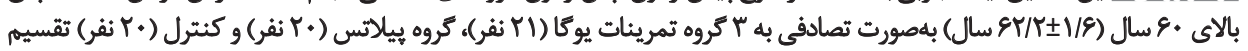

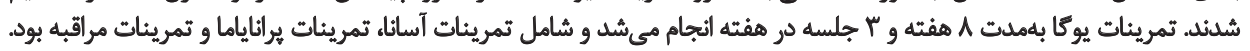

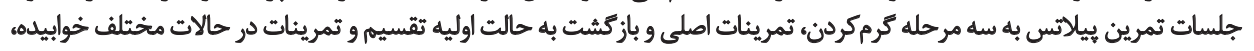

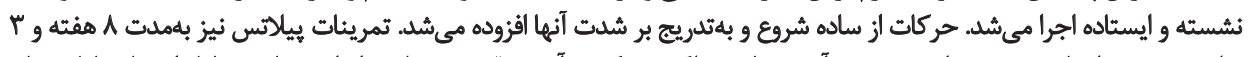

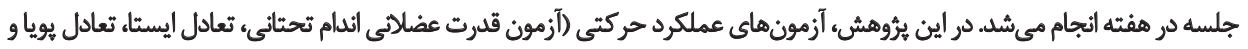

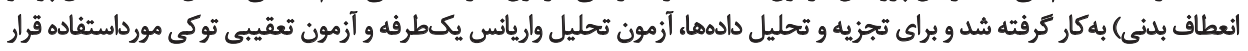

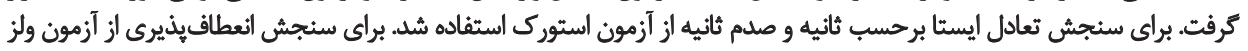

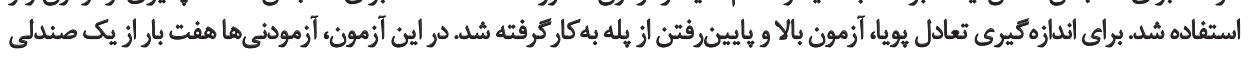

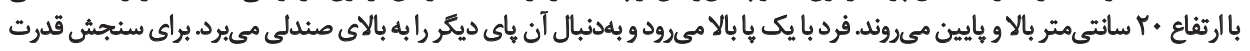

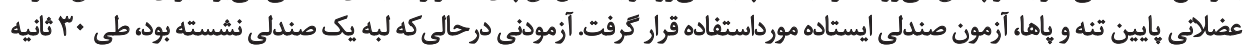

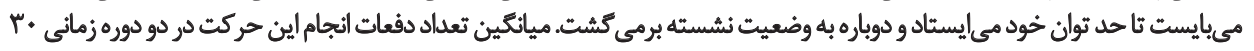

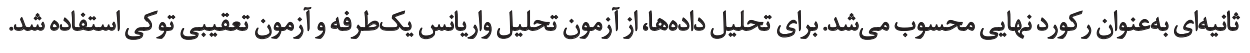

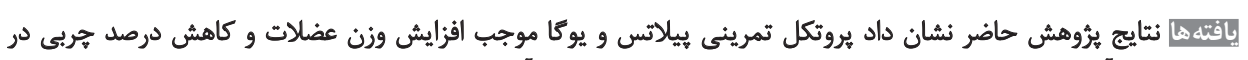

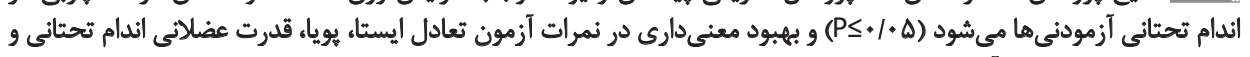

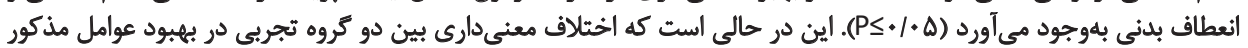

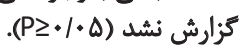

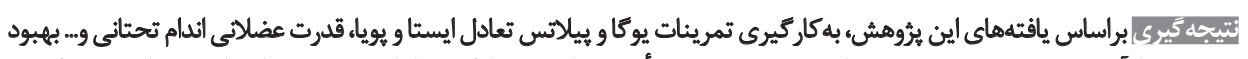

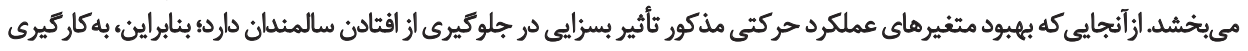
اين تمرينات براي سالمندان توصيه مي شئشود.

كليدوازوها:

عملكرد حركتى، انعطاف بدئى قدرت عضلانى، تعادل

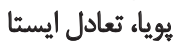

مي تواند سالمندان را با صدمات جدى ناشى از كاهش تعادل از مازئ

مقدمه

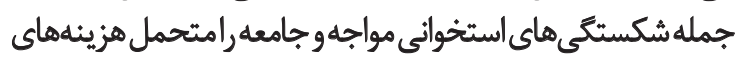

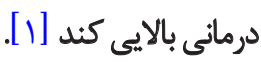

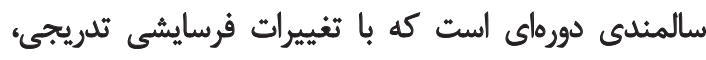

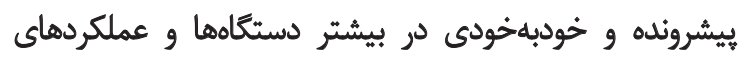
افتادن' يكى از مسائل مهمر و نتران كننداي است كه بيشتر

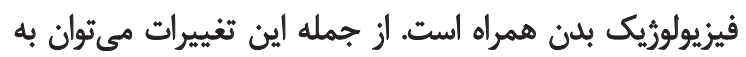
1. Falling تغييرات در دستكاههاى درگير در كنترل تعادل اشاره كرد كه

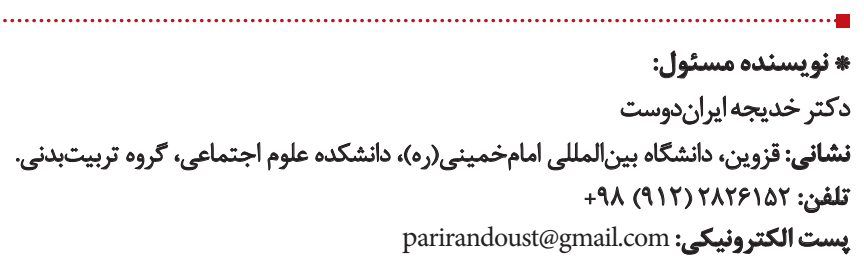


جسمى است كه در آن روى كنترل حركات، وضعيت قراركيرى بلن و تنفس تمركز مى شود. اين روش هاى تمريت تمرينى بر عواملى مانند انعطاف،

قدرت و تعادل تأكيد دارد [1 [1].

در اين يُروهش دليل بهكاركيرى روشهاى تمرينى آرامسازى

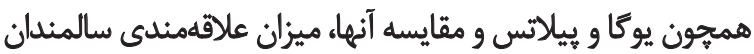

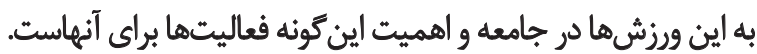

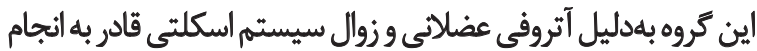
تمرينات هوازى و مقاومتى معمول نيستيند و در اين صورت آته احتمال

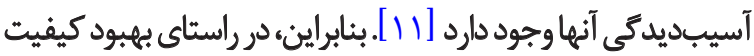

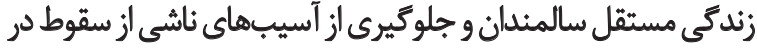

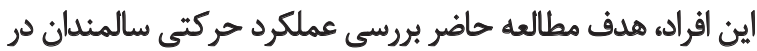
يكى تمرينات يوكاو بيلاتس است روش مطالعه

اين تحقيق نيمهتجربى با استفاده از طرح بيش آزمون-يس آزمون

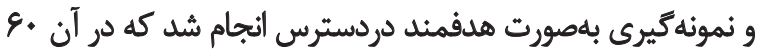

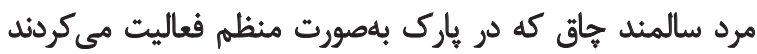

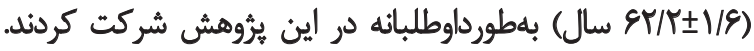

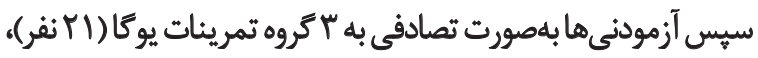

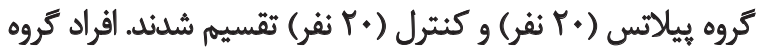

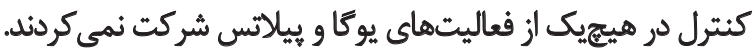

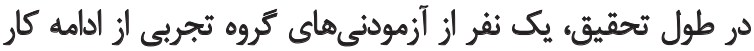

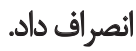

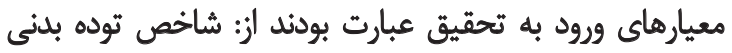

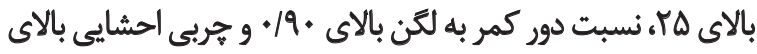

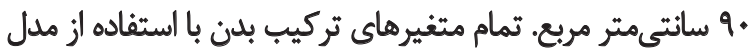

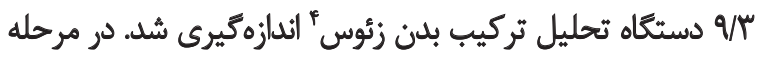

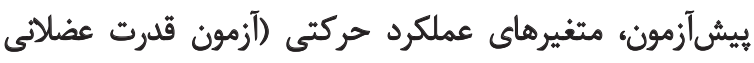

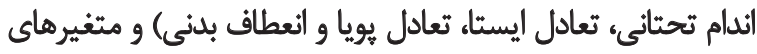

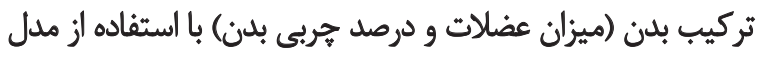

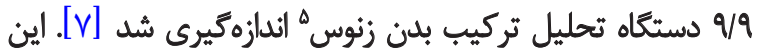

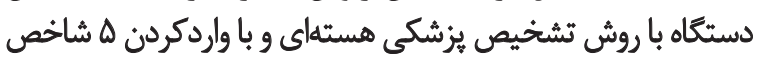

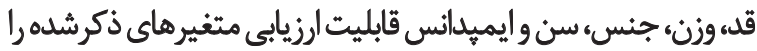

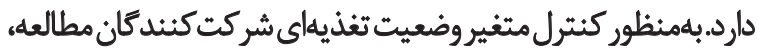

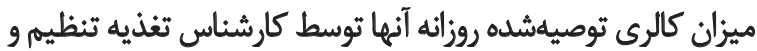

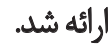

يروتكل تمرينى يوكاو ييلاتس توسط مربيان رسمى فدراسيونهاى

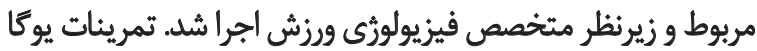

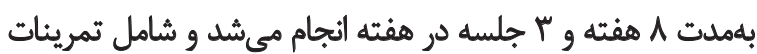
آسانا (حركات كششى، نرمشى بلهورتى كفه در تمام عضلات دركير

4. ZEUS 9.3 model

5. ZENUS 9.9 model

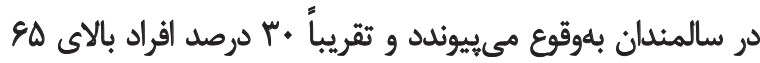

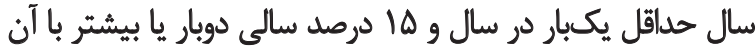

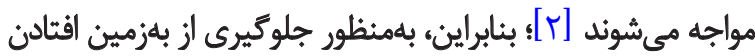

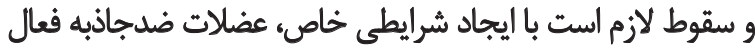

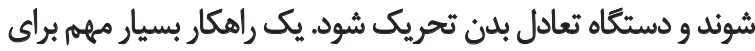

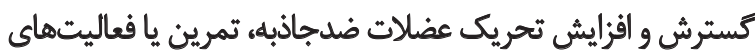

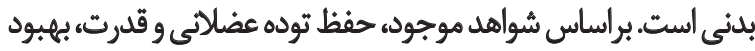

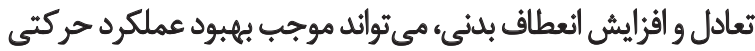

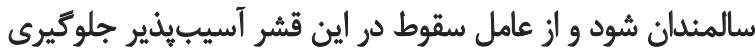
كند

امروزه بيشازئيش ثابت شده است كه افراد مسن مى توانئد با

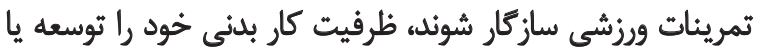

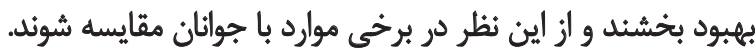

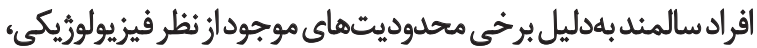

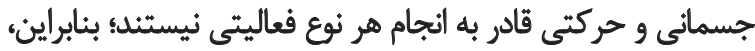

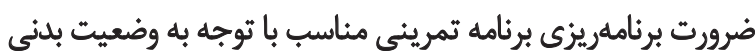

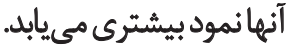

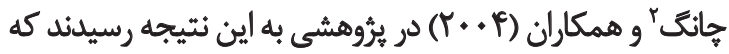

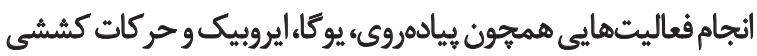

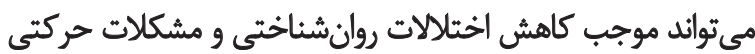

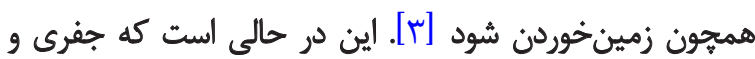

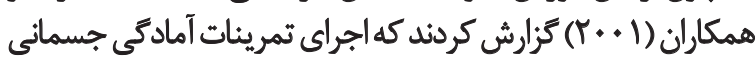

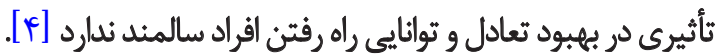

بسيارى ازمحققان راهرفتن و كنترل تعادل رابهاعنوان عوامل تعيين

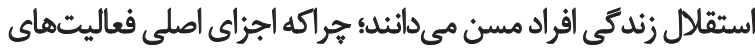

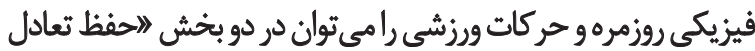

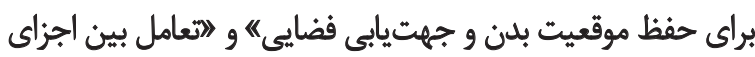

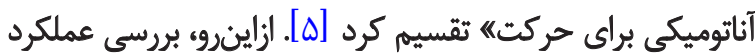

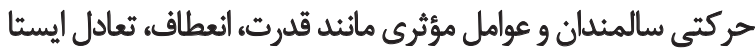
و يويا بسيارضرورى بهنظر ميرسد.

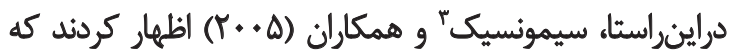

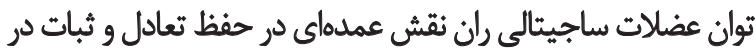

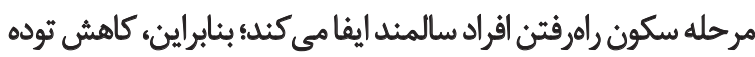

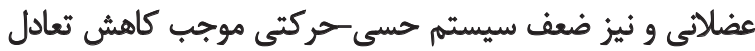

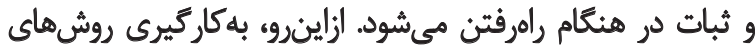

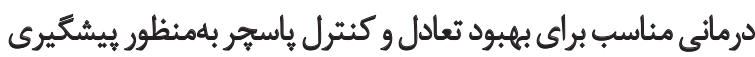

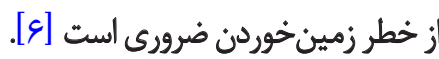

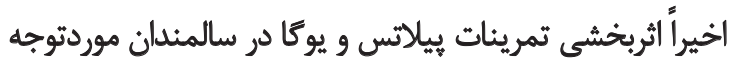

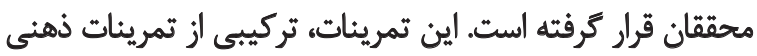

2. Chang

3. Simonsick 


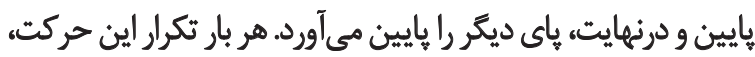

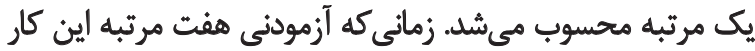

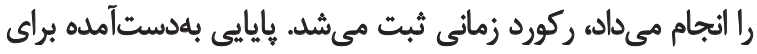

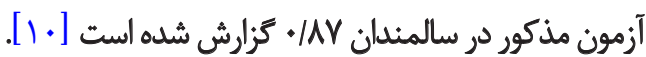
براى سنجش قدرت عضلاتى يايين تنه و واها، آزمون صندلى

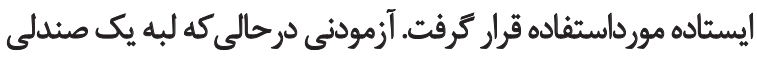

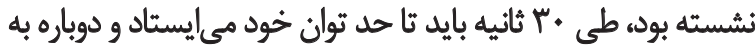

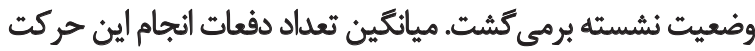

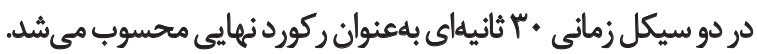

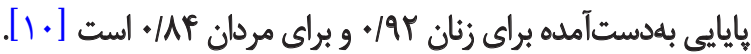

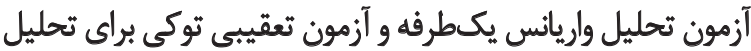

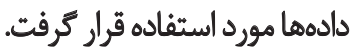

ياقثتهن

ميزان عضلات كل بدن، درصد جربي بلدن و عضله و جربى در

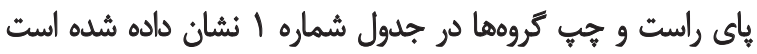

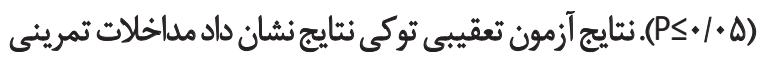

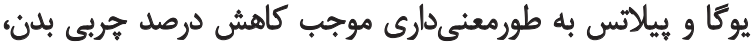

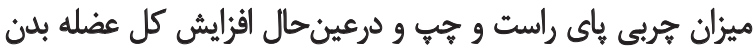

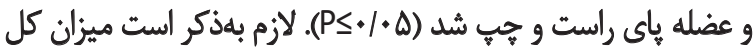

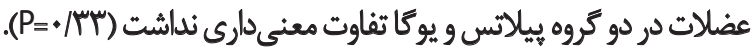

همان طور كه نتايج تحليل واريانس يكسرفه در جدول شماره ب نشان

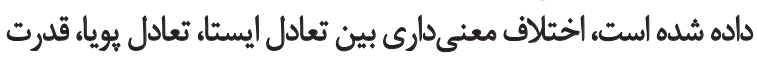

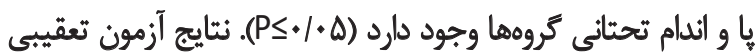

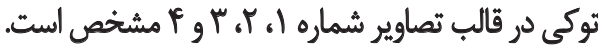

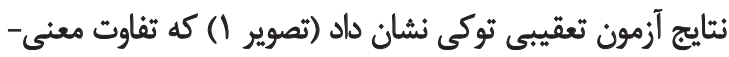

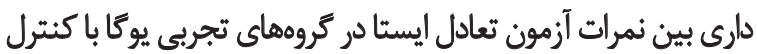

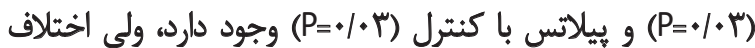

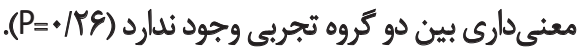
نتايج آزمون تعقيبى توكى نشان داد نمرات آزمون تعادل در كروه بروب

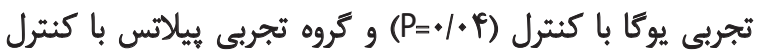

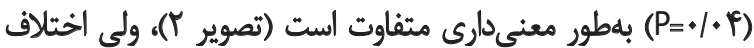

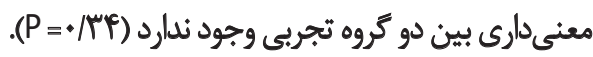

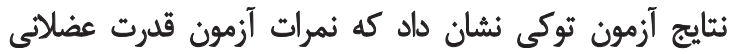

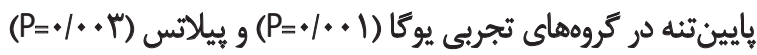

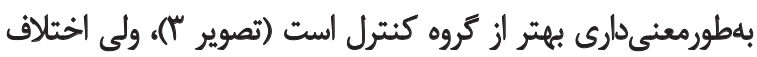

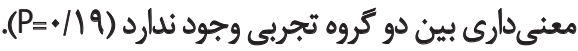
نتايج آزمون توكى نشان داد كه نمرات آزمون انعطاف بدنى در دران

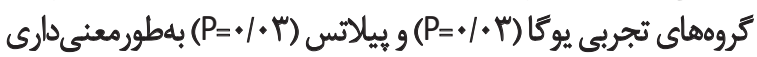

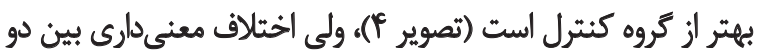

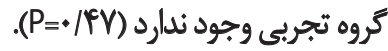

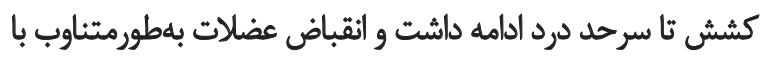

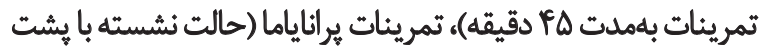

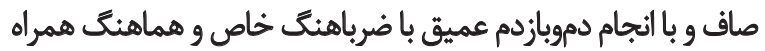

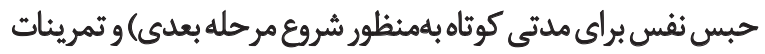

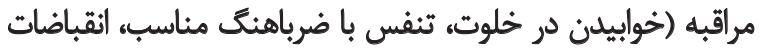

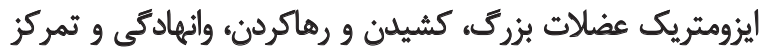

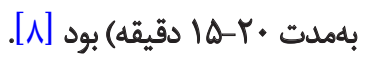

جلسات تمرين بيلاتس به سه مرحله كرمكردن، تمرينات اصلى و

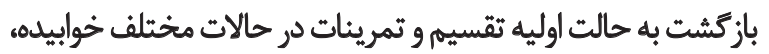

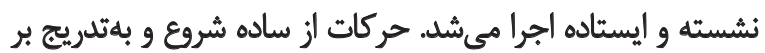

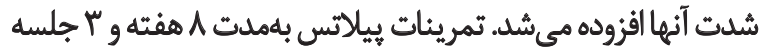

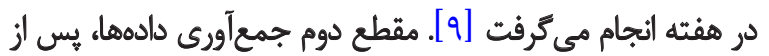

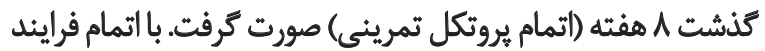

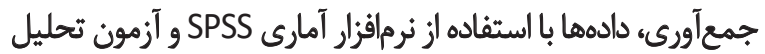

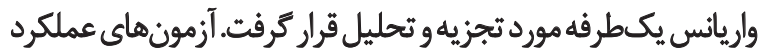
حركتى شامل آزمون قدرت عضلانى اندام تحتثاني، تعادل ايستا، تعادل يويا و انعطاف بدنى است.

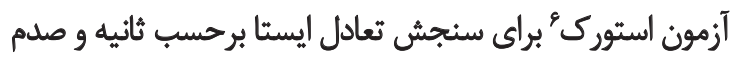

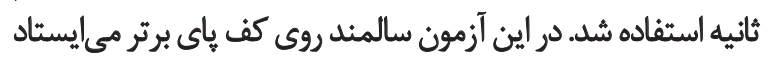

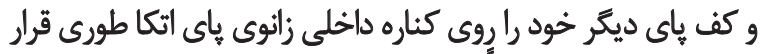

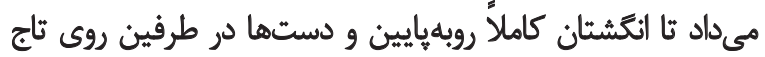

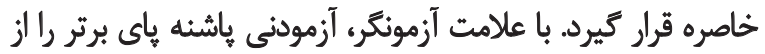

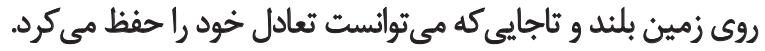

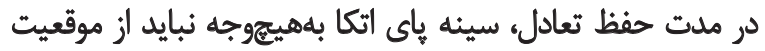

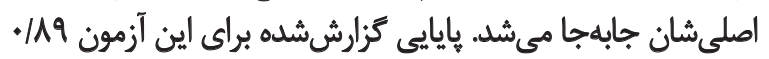

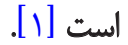

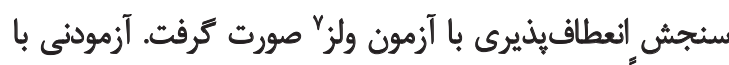

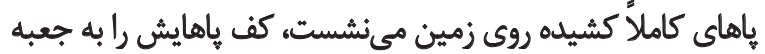

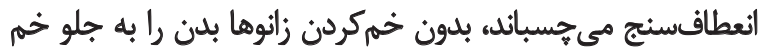

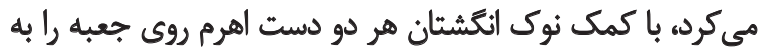

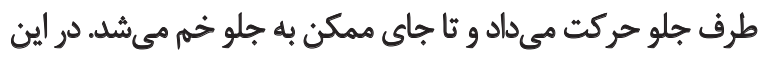

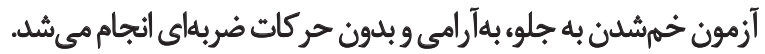

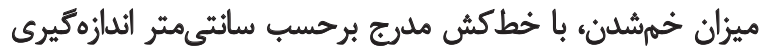

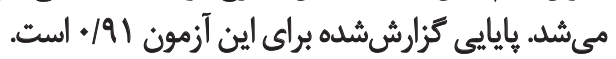

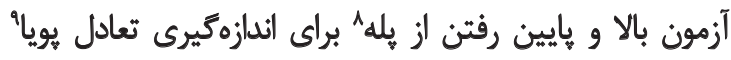

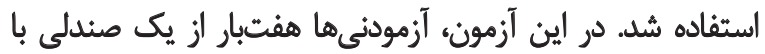

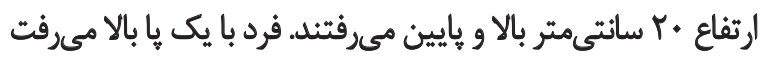

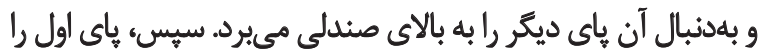

\section{Stork test}

7. Sit and reach

8. The timed up and down stair test

9. Dynamic balance 
جدول ا. مثايسه ويزٔكى هاى آنترويومتريك كروهها.

\begin{tabular}{|c|c|c|c|c|c|c|c|c|}
\hline \multirow{2}{*}{$\begin{array}{c}\text { P-value } \\
-\end{array}$} & \multicolumn{2}{|c|}{ كتترل } & \multicolumn{2}{|c|}{ بيلاتس } & \multicolumn{2}{|c|}{ يوكّا } & \multirow[b]{2}{*}{ ئروه } & \multirow[b]{2}{*}{ متغ } \\
\hline & $\frac{3}{3}$ & $\frac{3}{3}$ & $\frac{3}{3}$ & 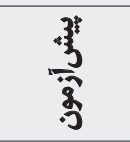 & $\frac{3}{3}$ & $\frac{3}{3}$ & & \\
\hline$+1+4$ & $r \Delta / T \pm V / r$ & $r \Delta / r \pm+/ \Lambda$ & $r W / \pm \backslash / \&$ & $r \Delta / / \pm) / 1$ & $m / q \pm 1 / 1$ & $r \Delta / q \pm 1 / r$ & كل بلن (كيلوكرم) & \\
\hline $.1 \cdot 1^{*}$ & $\varepsilon / \pm . / r$ & $\varepsilon / \mathbb{T} \pm \cdot / r$ & e/A.$\pm / r$ & $8 / \pm \cdot / r$ & $8 / q \pm . / r$ & $8 / K \pm \cdot / r$ & ياى راست (كيلوكرم) & $\frac{1}{3}$ \\
\hline$* r^{*}$ & $\varepsilon / \cdot \pm * / r$ & $8 / \pm+/ r$ & $g / N \pm * / r$ & $e / \cdot \pm \cdot / r$ & $s / N \pm \cdot R$ & \&/A & ياي هب (كيلوكرم) & \\
\hline $.1 . \mu$ & $r+/$ & $r+r$ & rNM & rit/ & TNE & MV & درصد جربي بلن (\%) & \\
\hline $.1+4$ & $m$ & 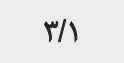 & $r / A$ & 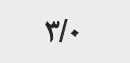 & $T / V$ & $r /$ & ياي راست (كيلوكرم) & $y$ \\
\hline $.1 \cdot 1^{*}$ & $m / M$ & $m$ & $r / V$ & $m$ & $r / A$ & $r /$ & هاي هب (كيلوكرم) & \\
\hline
\end{tabular}

L

جدول Y. مقايسه متغيرهاي تحقيق قبل و بعد از مداخله.

\begin{tabular}{|c|c|c|c|c|c|c|c|c|}
\hline \multirow{2}{*}{$\begin{array}{c}\text { P-value } \\
\qquad / \%{ }^{*}\end{array}$} & \multirow{2}{*}{$F$} & \multicolumn{2}{|c|}{ كتترل } & \multicolumn{2}{|c|}{ يبيلاتس } & \multicolumn{2}{|c|}{ يوكا } & \\
\hline & & يس أزمون & ينيش أزمون & يس أزمون & يبش أزمون & يس أزمون & بيش آزمون & \\
\hline$* /+r^{*}$ & elet & $\mid r / q \pm \cdot N$ & $\mid f / \& \pm . / \Delta$ & $\mid 8 / f \pm \cdot / 1$ & $\mid f / q \pm . / q$ & $\mid g / A \pm \cdot N$ & $\mid f / A \pm \cdot / q$ & تعادل ايستا \\
\hline $.1 \cdot r$ & $F / M$ & $\mid ه / r \pm 1 / r$ & $\mid \otimes / / \pm 1 / f$ & $\| / \Psi \pm 1 / F$ & $\mid \& / r \pm 1 / r$ & $11 / V \pm 1 / r$ & $|V / r| \pm 1 / r$ & تعادل يويا \\
\hline .1 .0 & $r / A)$ & 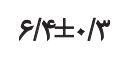 & $8 / T \pm . / \Delta$ & $9 / 9 \pm 1 / V$ & $8 / T \pm . / 9$ & $1 \cdot / 1 \pm 1 / r$ & $g / p \pm 1 / r$ & قدرت يا \\
\hline .1 .6 & $f / f$ & $r r / T \pm r / f$ & $r r / \pm T / r$ & $r F / q \pm 1 / q$ & $\mathrm{rr} / \cdot \pm \mathrm{N} / \mathrm{\Lambda}$ & $r \Delta / / \pm 1 / q$ & $r r / M \pm r / N$ & انعطاف بدنى \\
\hline
\end{tabular}

L

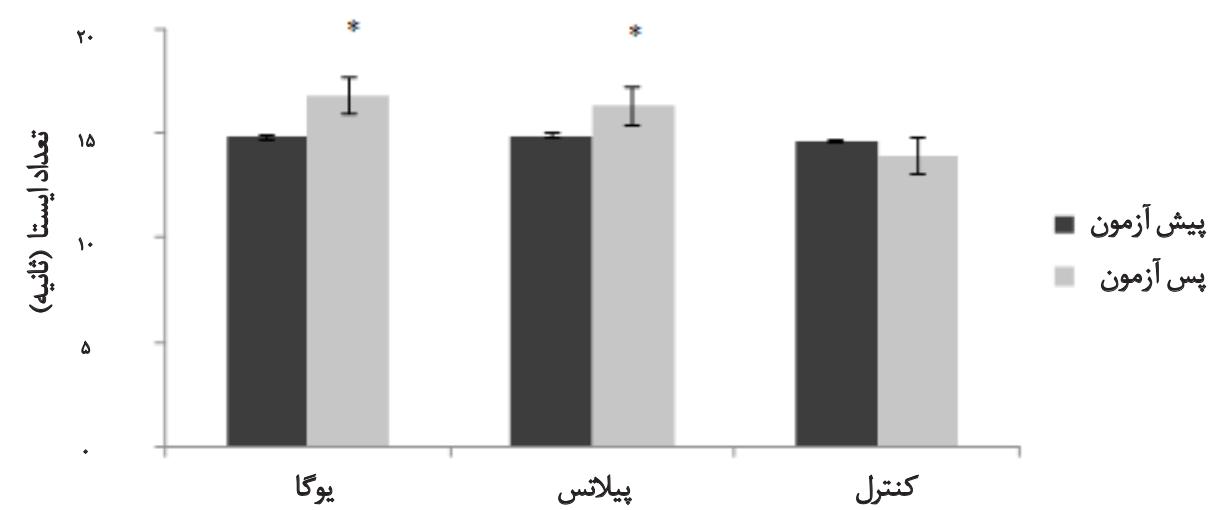

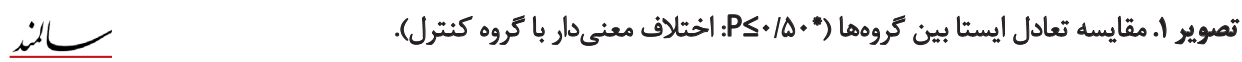



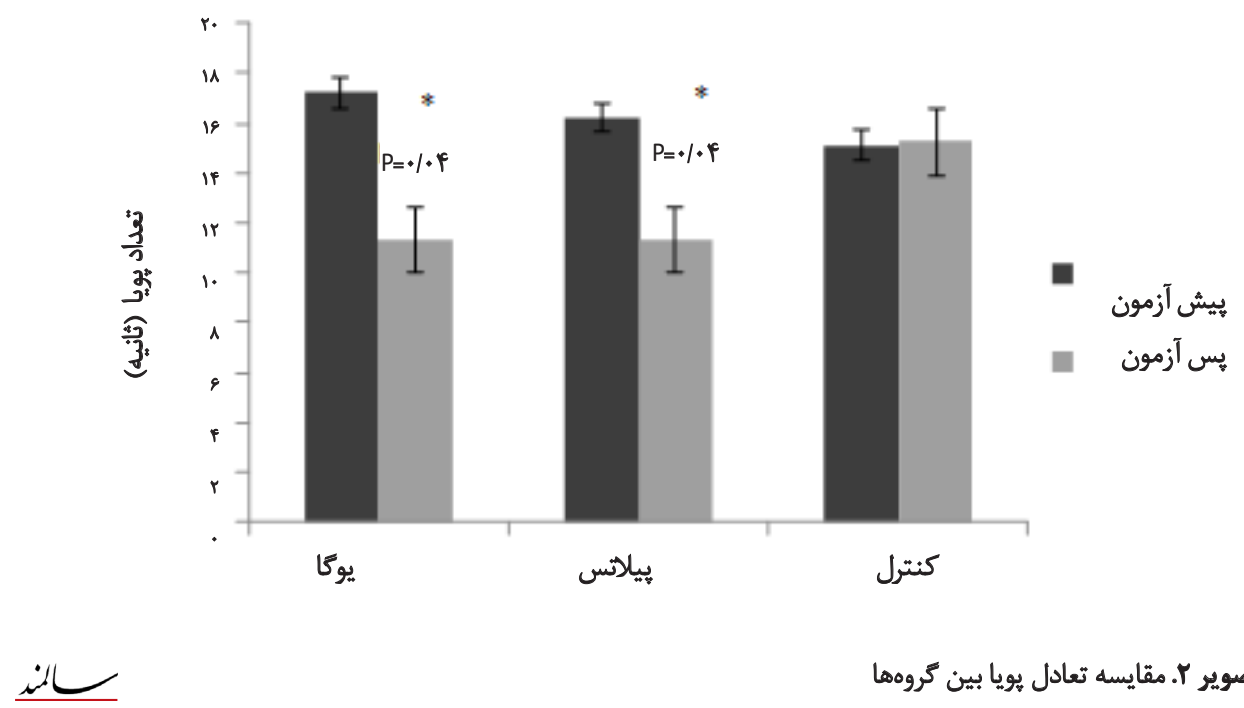

تصوير Y. مقايسه تعادل يويا بين كروهها

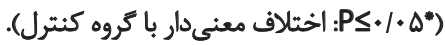

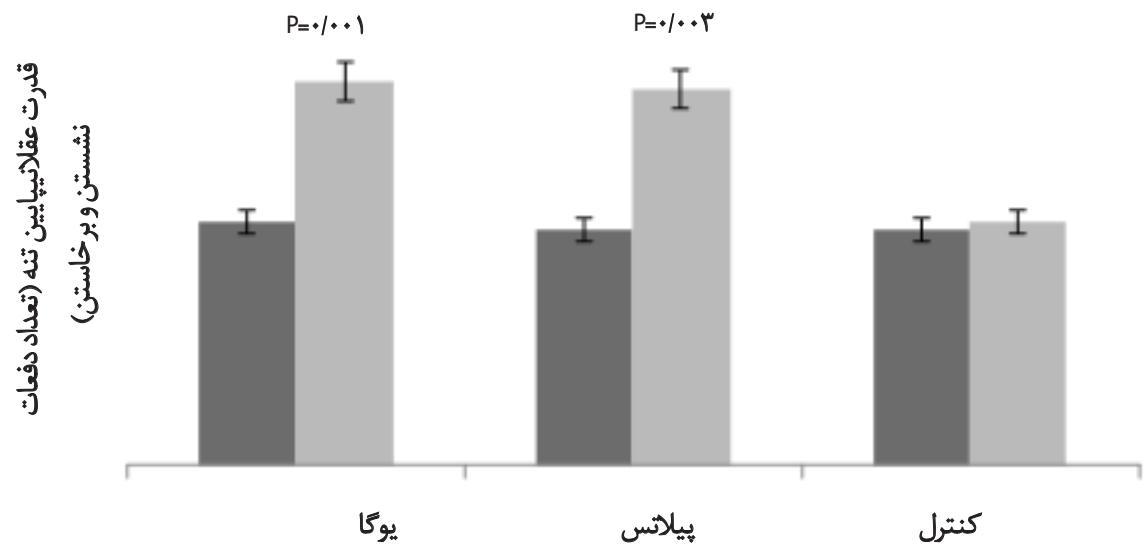

记

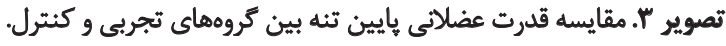

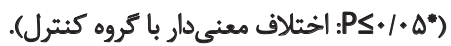

$\mathrm{P}=* 1 \cdot r \quad \mathrm{P}=* 1 \cdot r$

5
3
3
3
3
3
3
3
3
3

كالم

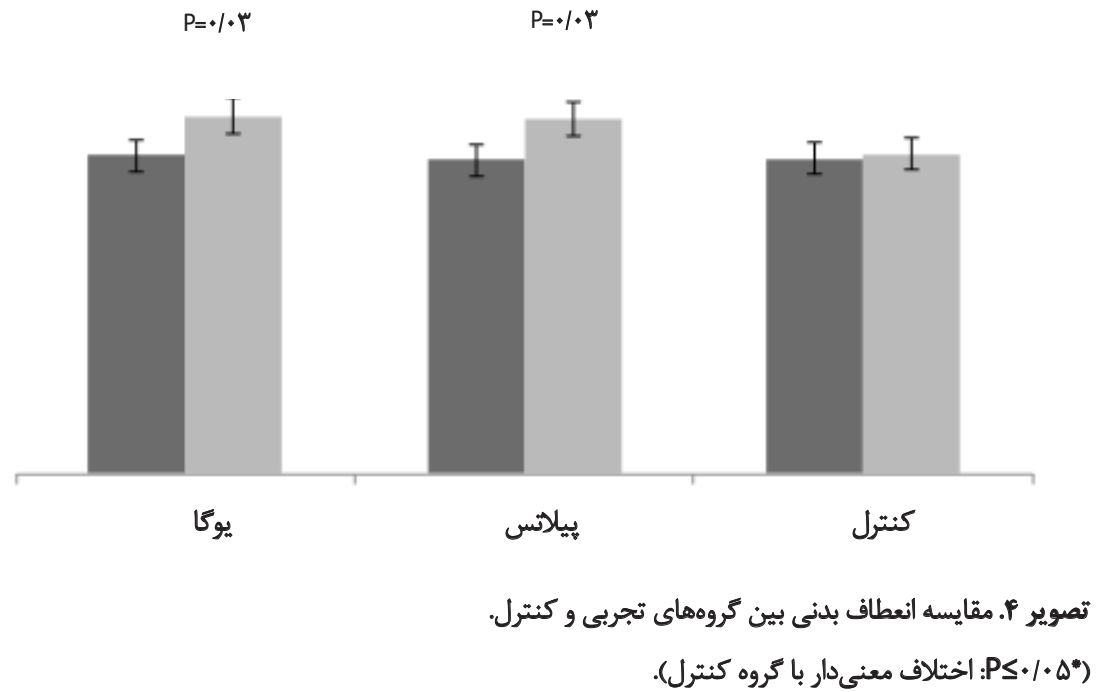


يكى از راهكارهاي مؤثر براى جلوكيرى از افتثادن سالمندان، افزايش

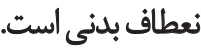

از طرف ديكر نتايج اين يرؤشش با تحقيق دبورال توركل و همكاران

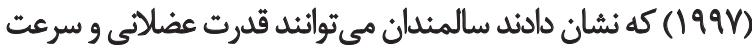

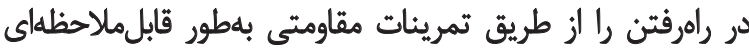

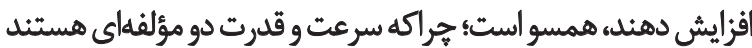

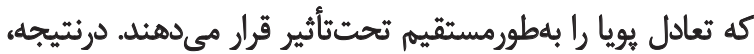

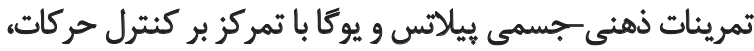

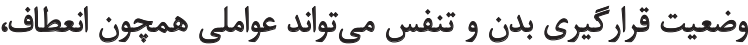

قدرت و تعادل را افزايش و عملكرد حركتى سالمئد را بهبود دهدي

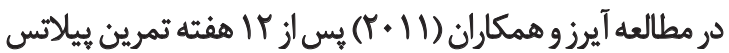
در زنان وهال، افزايش قدرت عضلانى تعادل يويا، زمان عكسان العمل

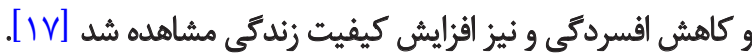

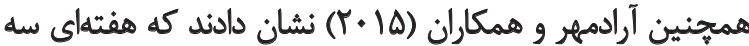

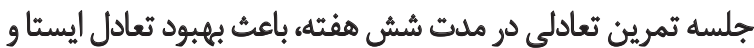

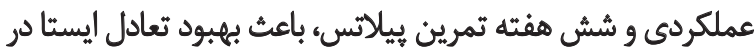
سالمندان مىشود كه هر دو مطالعه با تحقيق حاضر همخوانى دارند.

درحقيقت، تمرينات بيلاتس برمبناى هشت اصل شامل تنفس،

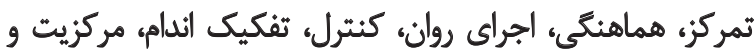

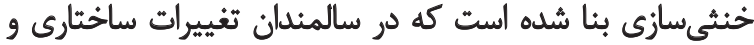

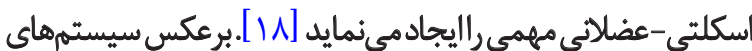

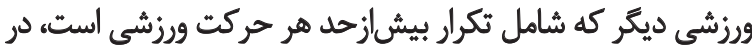

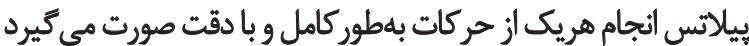

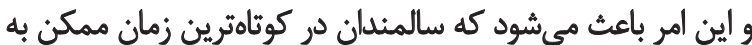

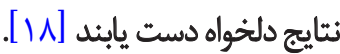

\section{نتيجهيرىنهايي}

ازأنجاييكه نتايج تحقيق نشان داد در دو كروه يوكأ و بيلاتس

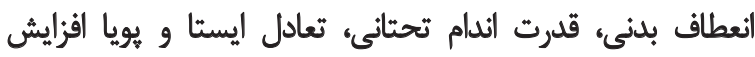

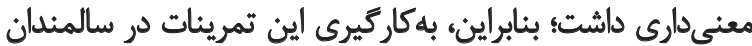

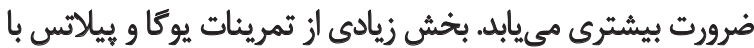

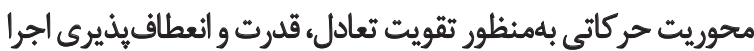

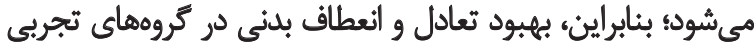

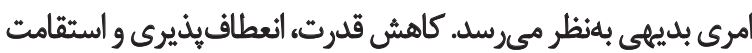

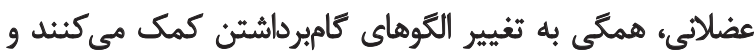

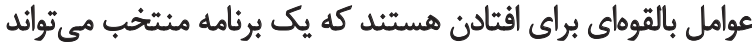
در :نين حوزهايي افت راجي جبران كند.

از محدوديتهاي مطالعه حاضر مى توان بهاه تك جنسيتى بوديون نمونه

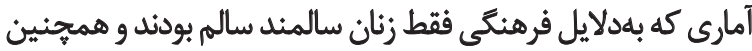

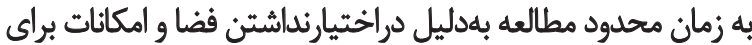

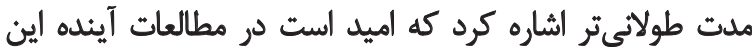
نكات در نظر كرفته شود. ازآنجاكه عوامل روانى مانيد افزايش ترسي،

هدف از اين تحقيق، بررسى عملكرد حركتى سالمندان بهدنبال

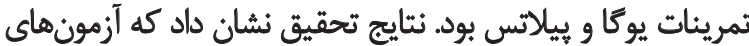

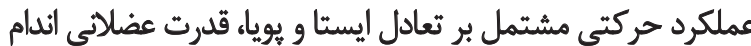

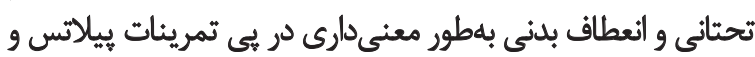

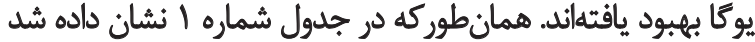

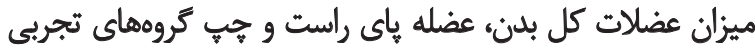

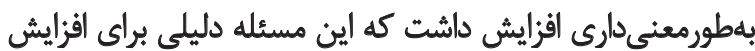

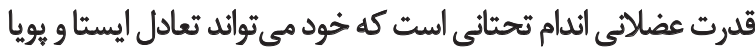

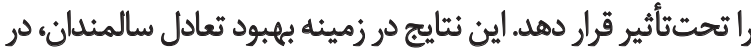

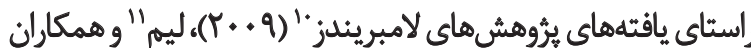

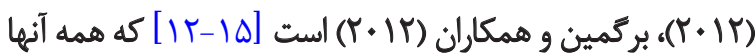

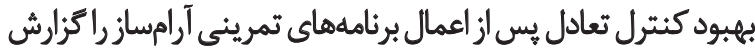

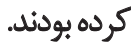

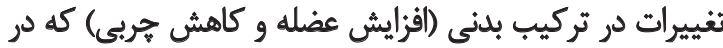

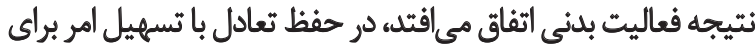

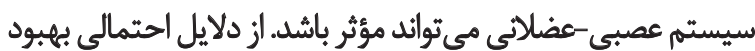

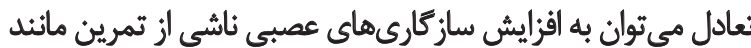

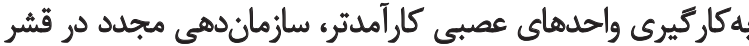

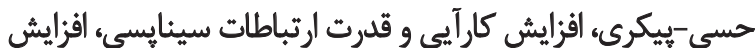

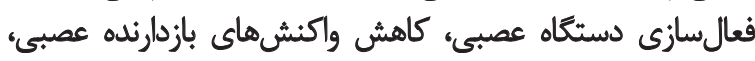

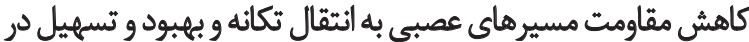
انتقال دروندادهاى هريك از حواس اشاره كرد. تمرينات يوكاو وييلاتس باتحريك حواس دركير در تعادل، شرايطى

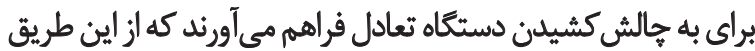

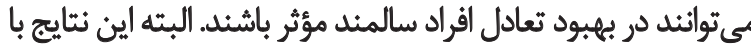

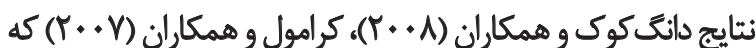

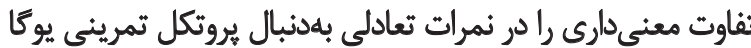

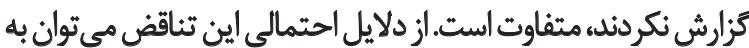

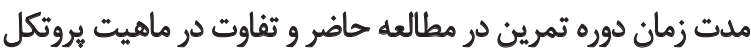

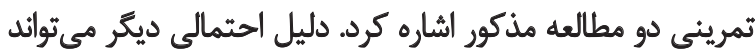

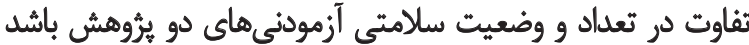

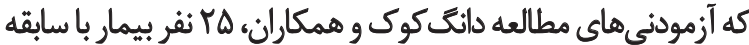

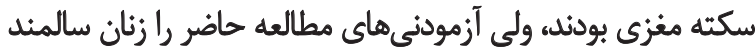

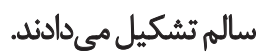

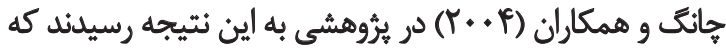

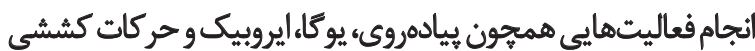

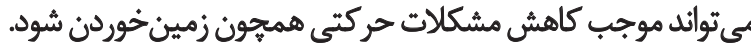
إزآنجاكه افزايش سن موجب كاهش انعطاف بدنى مى مشود؛ بنابراين،

10. Lambrinides

11. Lim 


\section{References}

[1] Sadeghi H, Norouzi HR, Karimi Asl A, Montazer MR. [Functional Training Program Effect on Static and Dynamic Balance in Male Able-bodied Elderly (Persian)]. Iranian Journal of Ageing. 2009; 3(2):565-571

[2] Tromp AM, Smit JH, Deeg DJ, Bouter LM, Lips PT. Predictors for falls and fractures in the Longitudinal Aging Study Amsterdam. Journal of Bone and Mineral Research. 1998; 13(12):1932-9.

[3] Chang JT, Morton SC, Rubenstein LZ, Mojica WA, Maglione M, Suttorp MJ, et al. Interventions for the prevention of falls in older adults: Systematic review and meta-analysis of randomised clinical trials. British Medical Journal. 2004; 328(7441):680-83.

[4] Schlicht J, Camaione DN, Owen SV. Effect of intense strength training on standing balance, walking speed, and sit-to-stand performance in older adults. Journals of Gerontology Series A: Biological Sciences and Medical Sciences. 2001; 56(5):281-6.

[5] Kimura T, Kobayashi H, Nakayama E, Hanaoka M. Effects of aging on gait patterns in the healthy elderly. Anthropological Science. 2007; 115(1):67-72.

[6] Simonsick EM, Guralnik JM, Volpato S, Balfour J, Fried LP. Just get out the door! Importance of walking outside the home for maintaining mobility: Findings from the women's health and aging study. Journal of the American Geriatrics Society. 2005; 53(2):198-203.

[7] Taheri M, Irandoust Kh. The effects of weight-loss induced aerobic exercise on Depressive Symptoms in the elderly. International Journal of Sport Studies. 2014; 3(5):295-298

[8] Javnbakht M, Kenari RH, Ghasemi M. Effects of yoga on depression and anxiety of women. Complementary Therapies in Clinical Practice. 2009; 15(2):102-4.

[9] Latey P. The Pilates method: History and philosophy. Journal of Bodywork and Movement Therapies. 2001; 5(4):275-82.

[10] Jones CJ, Rikli RE, Beam WC. A 30-s chair-stand test as a measure of lower body strength in community-residing older adults. Research Quarterly for Exercise and Sport. 1999; 70(2):113-9.

[11] Smith K, Smith E. Integrating pilates-based core strengthening into older adult fitness programs: Implications for practice. Topics in Geriatric Rehabilitation. 2005; 21(1):57-67.

[12] Lim YM, Hong GR. Effect of 16-week Kouk-Sun-Do exercise on physical fitness, emotional state, and immunoglobulin A in community-dwelling elders in Korea. Applied Nursing Research. 2010; 23(2):91-100.

[13] Ryushi T, Kumagai K, Hayase H, Abe T, Shibuya K, Ono A. Effect of resistive knee extension training on postural control measures in middle aged and elderly persons. Journal of Physiological Anthropology and Applied Human Science. 2000; 19(3):143-9.

[14] Hale LA, Waters D, Herbison P. A randomized controlled trial to investigate the effects of water-based exercise to improve falls risk and physical function in older adults with lower-extremity osteoarthritis. Archives of Physical Medicine and Rehabilitation. 2012; 93(1):27-34.

[15] Bergamin M, Zanuso S, Alvar BA, Ermolao A, Zaccaria M. Is water-based exercise training sufficient to improve physical fitness in the elderly? European Review of Aging and Physical Activity. 2012; 9(2):129-41.

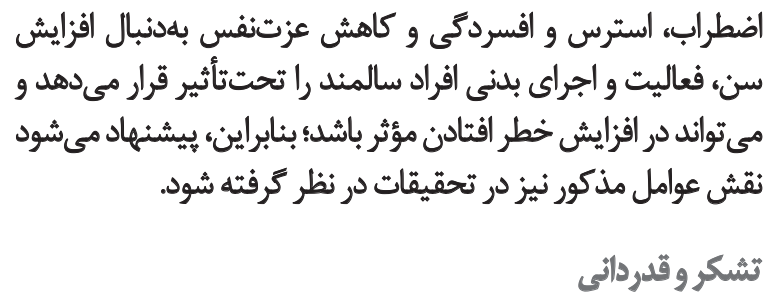


[16] Silva LE, Valim V, Pessanha AP, Oliveira LM, Myamoto S, Jones $\mathrm{A}$, et al. Hydrotherapy versus conventional land-based exercise for the management of patients with osteoarthritis of the knee: A randomized clinical trial. Physical Therapy. 2008; 88(1):12-21.

[17] Irez GB, Ozdemir RA, Evin R, Irez SG, Korkusuz F. Integrating Pilates exercise into an exercise program for $65+$ year-old women to reduce falls. Journal of Sports Science and Medicine. 2011; 10(1):105-11.

[18] Aradmehr M, Sagheeslami A, Ilbeigi S. [The effect of balance training and pilates on static and functional balanceof elderly men (Persian)]. Feyz. 2015; 18(6):571-577. 
